\title{
Characteristic Behaviors of Light Scattering from Polyelectrolyte in Dilute Solution Region
}

\author{
Masanori HARA and Akio NAKAJIMA \\ Department of Polymer Chemistry, Kyoto University, \\ Sakyo-ku, Kyoto 606, Japan.
}

(Received March 12, 1980)

\begin{abstract}
The characterstic properties of a polyelectrolyte in a dilute solution were investigated by light-scattering measurements using poly(sodium acrylate) with added salt. It was pointed out that (a) the second virial coefficient $\mathrm{A}_{2}$ of polyelectrolyte solution with added salt attained a high value as never before observed in neutral polymer solutions, (b) the intermolecular interference factor $Q(\theta)$ decreased remarkably with increasing scattered angle $\theta$, (c) the polyelectrolyte in the solution behaved as in a very good solvent. Even when the microsalt concentration had a relatively value as high as $0.1 \mathrm{M}$, a minimum point was found in the plot of dissymmetry ratio $Z_{45}$ against concentration $c$, and (d) $A_{2}$ was not proportional to $1 / c_{\mathrm{s}}^{1 / 2}$ but to $1 / c_{\mathrm{s}}$ in a microsalt concentration $\left(c_{\mathrm{s}}\right)$ range from $0.01 \mathrm{M}$ to $1 \mathrm{M}$ under the experimental conditions. These behavior characteristic are discussed in terms of a phase diagram.
\end{abstract}

KEY WORDS Polyelectrolyte / Light Scattering / Poly(sodium acrylate) / Second Virial Coefficient / Intermolecular Interference Factor / Dissymmetry Ratio / Phase Diagram /

So far, polyelectrolyte solution properties have been studied mainly by viscosity in aqueous solutions. ${ }^{1}$ This is because light scattering in aqueous solutions is difficult to measure and shows some anomalies, though light scattering is a major method for studying solution properties. The reasons for this are: (1) the scattered intensity is very week owing to destructive interference, and hence an instrument with very high sensitivity is required; (2) clarification of the solution is more difficult in the case of polyelectrolytes than for neutral polymers. Thus the number of systematic lightscattering studies for polyelectrolyte solutions are far less than those for neutral polymer solution.

In addition to those experimental difficulties, the following should be considered - since the polyelectrolyte has many charges along the chain, the long range force is effective and so the polyelectrolyte molecule behaves as in a very good solvent. Thus the light scattering from the polyelectrolyte should be measured at a very dilute concentration. However, the concentrations so far measured $^{2-5}$ ranged from $5 \times 10^{-4}$ to $3 \times 10^{-3}$ $\mathrm{g} \mathrm{cm}^{-3}$. Such a concentration range corresponds to a dilute solution range for neutral polymers, but is not sufficiently low for investigating polyelectrolyte solutions.

The lack of light-scattering data for polyelectrolyte solutions is attributed not only to the experimental difficulties involved but also to a basic character of the solution. In this paper, we are concerned with a dilute aqueous solution of polyelectrolyte and investigate its characteristic behavior in regard to light scattering. The concentration dependence ${ }^{8}$ of the dissymmetry ratio $Z_{45}$, and microsalt concentration dependence ${ }^{6,7}$ of second virial coefficient $A_{2}$ are also discussed. Also, in a subsequent paper, we will discuss the anomalies involved in a polyelectrolyte solution and compare them with a very high-molecular-weight neutral polymer solution.

\section{Materials}

\section{EXPERIMENTAL}

Poly(acrylic acid) (PAA), purchased from Polyscience Co. in the form of a $25 \%$ aqueous solution, was purified and fractionated by repeating three times dissolution in $0.4 \mathrm{M} \mathrm{NaOH}$ aqueous solution and precipitation with methanol. The polymer obtained as sodium salt was dissolved in water 


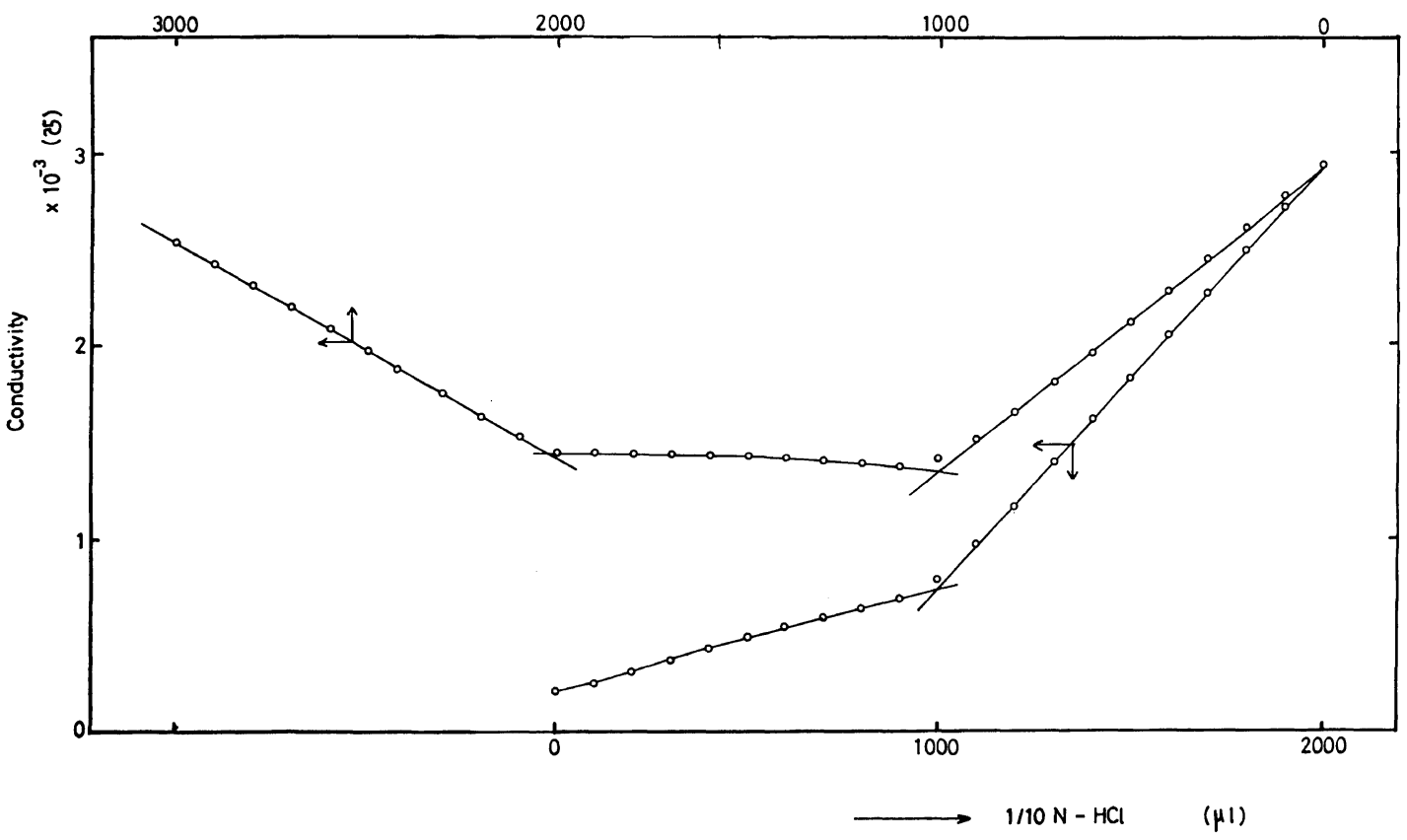

Figure 1. Conductometric titration of PAA with $0.1 \mathrm{~N}-\mathrm{HCl}$ and $0.1 \mathrm{~N}-\mathrm{NaOH}$.

treated by duplicated distillation in an all-glass still. The solution obtained was used as the stock solution. The $\mathrm{COOH}$ content of the PAA sample was determined as $96 \%$ by conductometric titration as shown in Figure 1 using a Yanagimoto Conductometry Outfit Model MY-8. Polystyrenes were molecular-weight standard samples purchased from Pressure Chemical Co. $\left(M_{w}=1.1 \times 10^{5}\right.$ and $3.9 \times 10^{5} ; M_{w} / M_{n}$ is less than 1.06 and 1.10 , respectively). Toluene and benzene for spectroscopy were used after distillation.

\section{Light-Scattering Measurements}

The scattered intensity was measured by using a Fica 50 photogoniometer (SOFICA France) at 11 different angles ranging from 30 to $150^{\circ}$ with light of $546 \mathrm{~nm}$ in wavelength. The positions of the analizer and polarizer were checked by measuring $V_{\mathrm{v}}(\theta)$, $H_{\mathrm{v}}(\theta), V_{\mathrm{h}}(\theta)$, and $H_{\mathrm{h}}(\theta)$ of benzene as shown in Figure 2, where $V_{\mathrm{v}}(\theta)$ and $H_{\mathrm{v}}(\theta)$ represent the vertical and horizontal components of the scattered light when the incident light is vertically polarized at angle $\theta$, and $H_{\mathrm{h}}(\theta)$, and $V_{\mathrm{h}}(\theta)$ vice versa. Figure 2

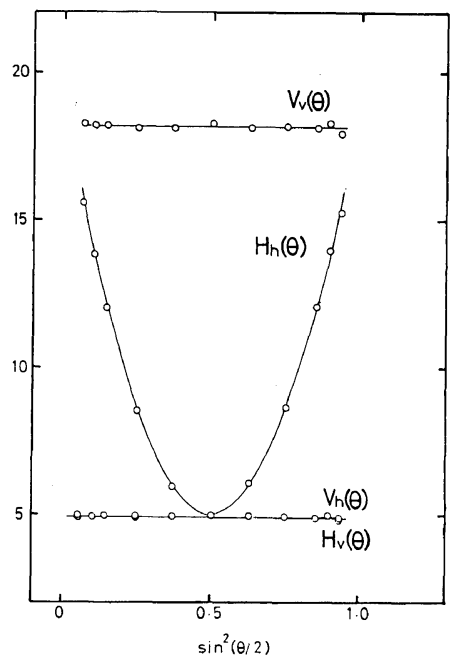

Figure 2. Plots of reduced scattered intensity of polarized light against $\sin ^{2}(\theta / 2)$ for benzene at $25^{\circ} \mathrm{C}$.

satisfies the requirements for accuracy of measurement; that is, the $V_{v}, H_{v}$, and $V_{\mathrm{h}}$ components are constant irrespective of the angle, and the $H_{\mathrm{h}}$ 
component, which coincides with the $H_{\mathrm{v}}$ and $V_{\mathrm{h}}$ components at $\theta=\pi / 2$, is symmetrical about $\theta=\pi / 2$. All measurements were performed with polarized light in order to check the optical anisotropy, i.e., $V_{\mathrm{v}}(\theta)$ and $V_{\mathrm{h}}\left(90^{\circ}\right)$ were measured and if necessary ${ }^{9}$ $4 V_{\mathrm{h}}\left(90^{\circ}\right) / 3$ was subtracted from $V_{\mathrm{v}}(\theta)$.

The optical clarification of the solution was carried out by centrifugation at $50,000-80,000 \mathrm{~g}$ for $2-3$ hours using a Marusan centrifuge $50 \mathrm{~S}$. The solvent was then transferred gradually from the centrifuge tube into the light-scattering cell using a special pipette by a gradual flow of water from a reservoir in order not to disturb the solution.,10 Also the solution was transferred in the same manner from the centrifuge tube into the special burette by which the solution was transferred into the cell for each measurement.

Temperatures were kept at $25 \pm 0.1^{\circ} \mathrm{C}$ and $15 \pm 0.5^{\circ} \mathrm{C}$ by operating a heater situated within the Fica 50 and circulating the cooling water. The measuring cell was an optically ground and polished one for aqueous solutions and a special Teflon cap with a small hole was placed on the cell in order to prevent dust from entering. All glasswares (cell, pipette, and burette) were cleaned for 2 hours with condensed acetone vapor using a Thurmond-type instrument. ${ }^{11}$

In order to determine the calibration constant, $U_{\mathrm{u}}\left(90^{\circ}\right)=16.0 \times 10^{-6}$ was used. $U_{\mathrm{u}}\left(90^{\circ}\right)$ represents the Rayleigh ratio of benzene at $25^{\circ} \mathrm{C}$ and $546 \mathrm{~nm}$ when neither the polarizer nor the analizer was used. ${ }^{12,13}$ After the usual adjustments (to make sure that the stray light is negligible and that the instruments have long term stability), the light scattering of the polystyrene standard samples were measured in toluene. The molecular weight obtained agreed with the nominal value ${ }^{14}$ within $1 \%$, and the value of $A_{2}$ and $\left\langle S^{2}\right\rangle^{1 / 2}$ were consistent with the data of Berry. ${ }^{15}$

\section{Refractive Index Increment}

The refractive index increment with concentration, $\mathrm{d} n / \mathrm{d} c$, was measured using a Uniongiken RM-101 differential refractometer. A special Bricetype cell incorporated with sockets and Teflon stoppers was used in order to prevent any mixing of solution with solvent. The calibration constant was determined using a $\mathrm{KCl}$ solution. To check the reliability of the instrument, $\mathrm{d} n / \mathrm{d} c$ value was measured for polystyrene in toluene. The obtained value 0.110 agreed with the literature value. ${ }^{16,17}$

\section{Dialysis}

The dialyzer assembly shown in Figure 3 was constructed according to the original design of professor A. Takahashi. The outer case (a), the dialyzer cell ((b), (d)) and the screws were made of poly(methyl methacrylate) resin. The solution was introduced into the dialyzer cell (b), and the solvent was kept in (a). The outer case installed the dialyzer cell was placed in a thermostat of $25 \pm 0.01^{\circ} \mathrm{C}$. The

$$
\text { dialyzer }
$$
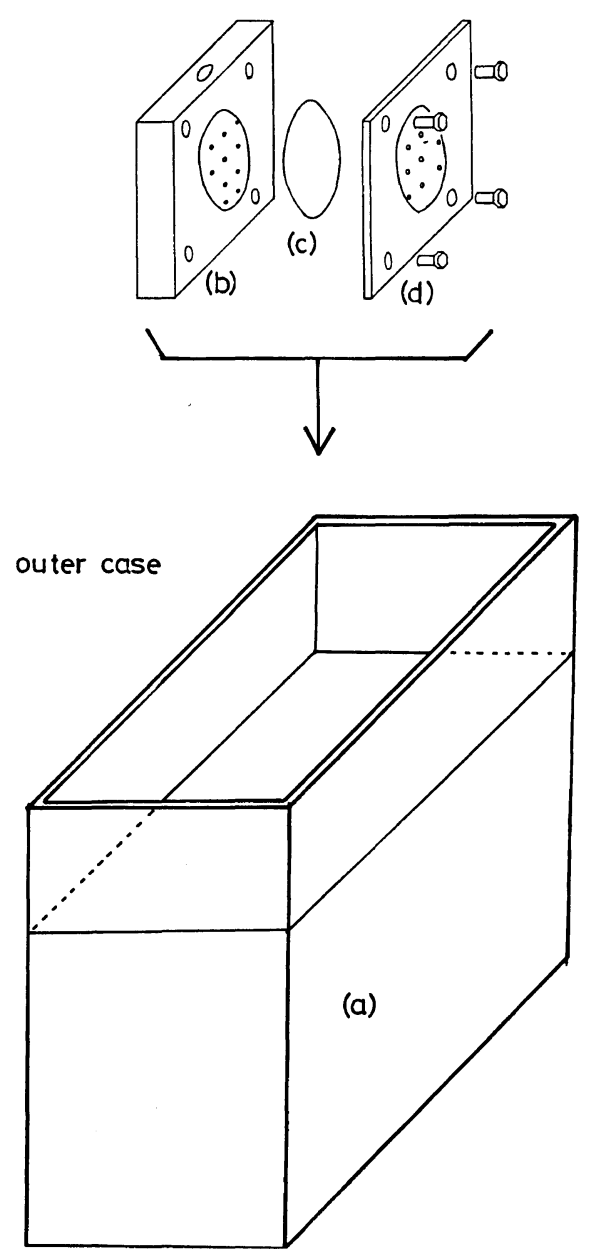

Figure 3. Dialyzer assembly: (a) outer-case containing solvent; (b) dialyzer cell; (c) membrane; (d) membrane holder. 
solvent was renewed each day, and the dialyzer was shaken once per $1-2$ hours. The membrane used was RC-51 (regenerated cellulose) purchased from Carl Schleicher \& Schull.

\section{Data Analysis}

The following two points were considered in analyzing the data:

(1) The reflection correction ${ }^{18,19}$ at the glasswater interface was made by eq 1 ,

$$
\begin{aligned}
R_{\theta} & =\frac{1}{t_{\mathrm{s}}^{2}\left(1-4 f_{\mathrm{s}}^{2}\right)}\left(R_{\theta}^{\prime}-2 f_{\mathrm{s}} R_{\pi-\theta}^{\prime}\right) \\
& =\frac{1}{0.9949}\left(R_{\theta}^{\prime}-0.0051 R_{\pi-\theta}^{\prime}\right)
\end{aligned}
$$

where $R_{\theta}^{\prime}$ and $R_{\theta}$ are the reduced scattered intensities at the angle $\theta$ before and after correction, respectively, $f_{\mathrm{s}}$ is the fraction of incident light reflected at the water-glass interface, and $t_{\mathrm{s}}$ is equal to $1-f_{\mathrm{s}}$. Because the dissymmetry of our system is less than 5 at $30^{\circ}$, the reflection correction comes to $2-3 \%$ at most and the correction by calculation is sufficient. By this correction, the curved line at higher angles became straight.

(2) The data analysis for the multicomponent system was performed by the following equations. ${ }^{20}$

$$
\begin{array}{r}
{\left[K c / R_{\theta}\right]_{\theta=0}=1 / M_{\mathrm{ap}}+2 A_{2, \mathrm{ap}} c+\cdots} \\
\quad(\text { conventional method) } \\
{\left[K^{*} c / R_{\theta}^{*}\right]_{\theta=0}=1 / M+2\left(A_{2}+\delta\right) c+\cdots} \\
\quad(\text { dialysis method) }
\end{array}
$$

$$
\left[K^{*} c / R_{\theta}\right]_{\theta=0}=1 / M+2\left(A_{2}+\delta+\Delta / M\right) c+\cdots
$$

$$
\begin{aligned}
{\left[K c / R_{\theta}\right]_{c=0} } & =\left(1 / M_{\mathrm{ap}}\right)\left[1+\left(16 \pi^{2} / 3 \lambda^{\prime 2}\right)\right. \\
& \left.\times\left\langle S^{2}\right\rangle \sin ^{2}(\theta / 2)+\cdots\right]
\end{aligned}
$$

(conventional method)

where $\lambda^{\prime}$ is the wavelength of the light in solution, $\delta$ and $\Delta$ are the thermodynamic parameters defined in the original paper, ${ }^{20}$ and $K$ is the scattering constant defined by

$$
K=\frac{4 \pi^{2} n_{0}^{2}(\mathrm{~d} n / \mathrm{d} c)^{2}}{N_{\mathrm{A}} \lambda_{0}^{4}}
$$

in which $\lambda_{0}$ is the wavelength in vacuo, $n_{0}$ is the refractive index of the solvent, and $N_{\mathrm{A}}$ is
Avogadro's number. The subscript ap denotes the apparent value and the asterisk $*$ denotes the value for dialyzed sample. These equations were derived by the distribution function theory ${ }^{25}$ and were used in order to analyze the mixed solvent system. However, as is the case of fluctuation theory, ${ }^{21}$ these equations can be used to analyze the polyelectrolyte-microsalt system. Because $\delta$ is usually negligible compared with $A_{2}$, the real molecular weight $M$ and the real second virial coefficient $A_{2}$ are obtained from eq 4 under the condition that $A_{2} \gg \Delta / M$ by measuring $\mathrm{d} n / \mathrm{d} c$ of dialized sample and the $R_{\theta}$ of undialyzed sample. Also from eq 5 , the real radius of gyration $\left\langle S^{2}\right\rangle^{1 / 2}$ is obtained from measurements on the undialyzed sample.

\section{RESULTS AND DISCUSSION}

\section{Zimm Plot}

A Zimm plot ${ }^{25}$ for PAA in a $1.5 \mathrm{M} \mathrm{NaBr}$ aqueous solution at $15^{\circ} \mathrm{C}$ is shown in Figure 4, from which the molecular weight was obtained as $5.0 \times 10^{5}$. Owing to the polydispersity of the PAA sample, the Zimm plot is convex slightly upward at smaller angles. The data at higher angles lead to the number-average molecular weight $M_{n}^{22}$; thus $M_{w} / M_{n}$ was estimated as 2.2. By using the procedure of Benoit et al ${ }^{23}$ with $\varepsilon=0.35$, it was found that this value agrees with the value 2.1 obtained for PAA in a $0.1 \mathrm{M} \mathrm{NaCl}$ aqueous solution.

Zimm plots for the PAA-NaCl systems are shown in Figure 5. The reproducibility of the data examined from measurements repeated 3-5 times was within $1-3 \%$. The characteristic features of these curves are as follows: (1) the angle dependence of the reciprocal intensity function $c / R_{\theta}$ is normal below a critical concentration, but above this concentration, it becomes convex upward markedly at smaller angles, (2) though the concentration dependence curve of $c / R_{\theta}$ is normal below the critical concentration, i.e., concave upward, it becomes convex upward above this concentration. The lower the microsalt concentration $c_{\mathrm{s}}$ is, the more remarkable are these anomalies. We will discuss these anomalies in the next paper, and so consequently in this paper we concerned mainly with the behavior below the critical concentration.

Because a dilute polyelectrolyte solution behaves as a very good solvent system, Flory's equation ${ }^{24}$ 
Light Scattering from Polyelectrolyte in Dilute Solution

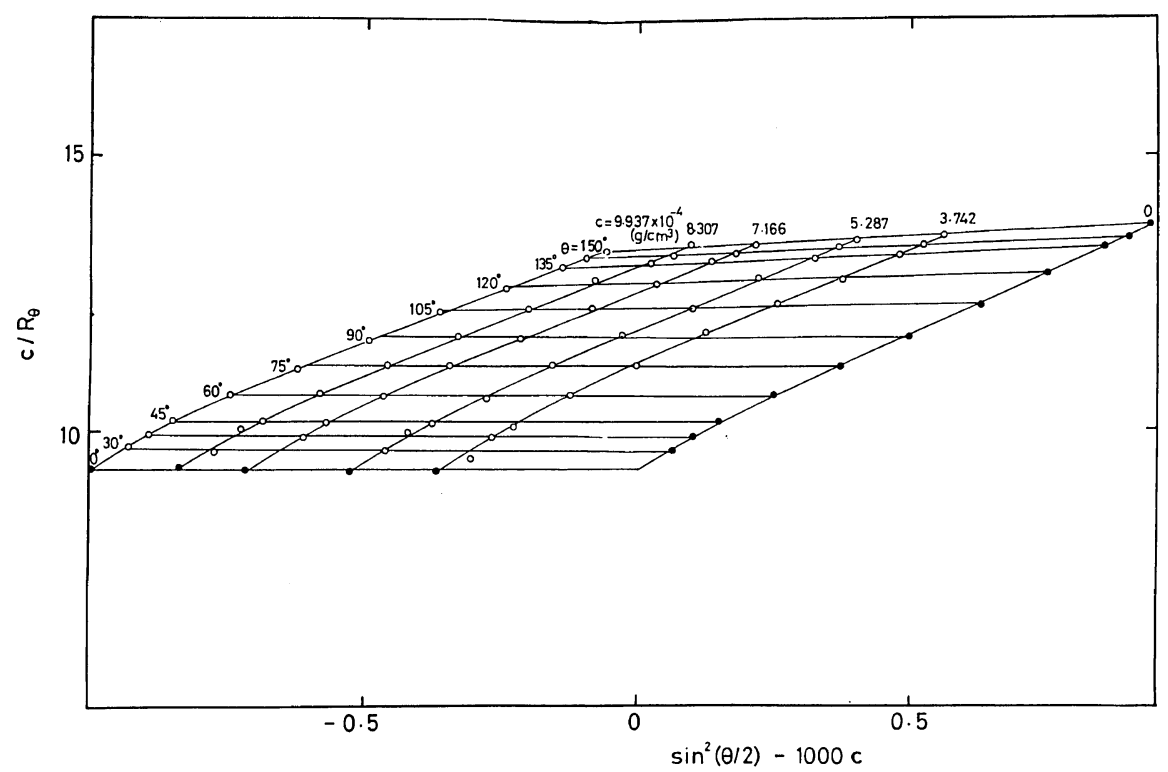

Figure 4. Zimm plot for PAA-1.5 M NaBr system at $15^{\circ} \mathrm{C}$.

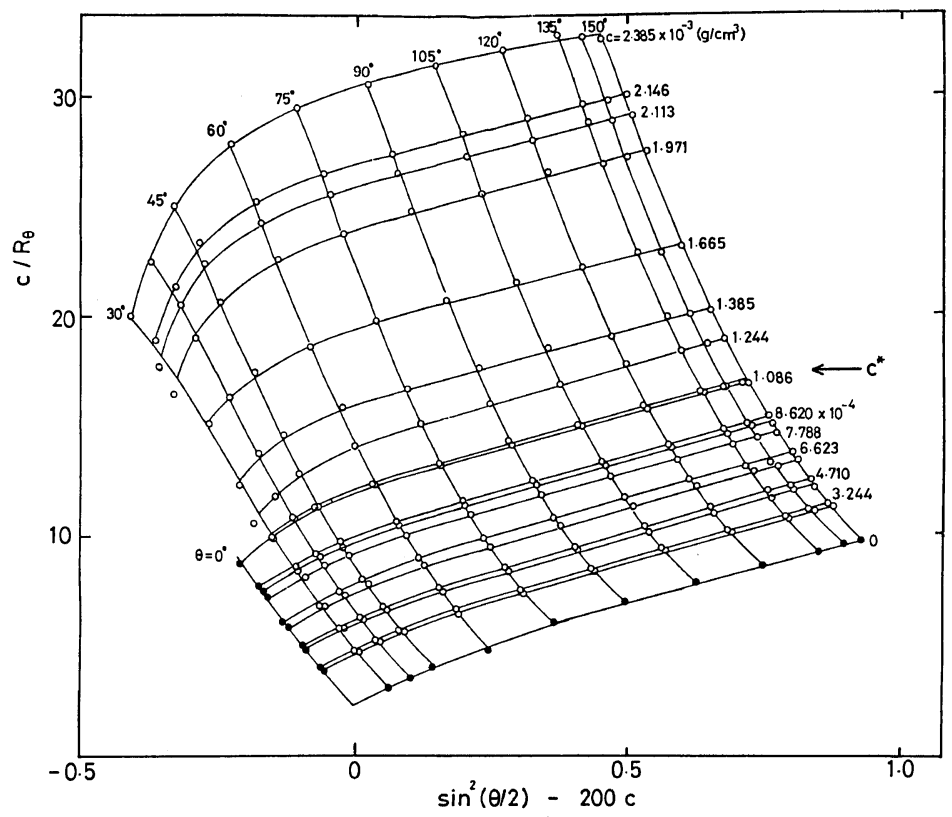

Figure 5(a). Zimm plot for PAA-0.1 M NaCl system at $25^{\circ} \mathrm{C}$.

(eq 6) can be used well, instead of Zimm's equation $^{25}$ which is derived according to the single contact approximation and is only appropriate for $\frac{K c}{R_{\theta}}=\frac{1}{M P(\theta)}+2 A_{2} Q(\theta) c+\cdots$ poor solvent systems, 


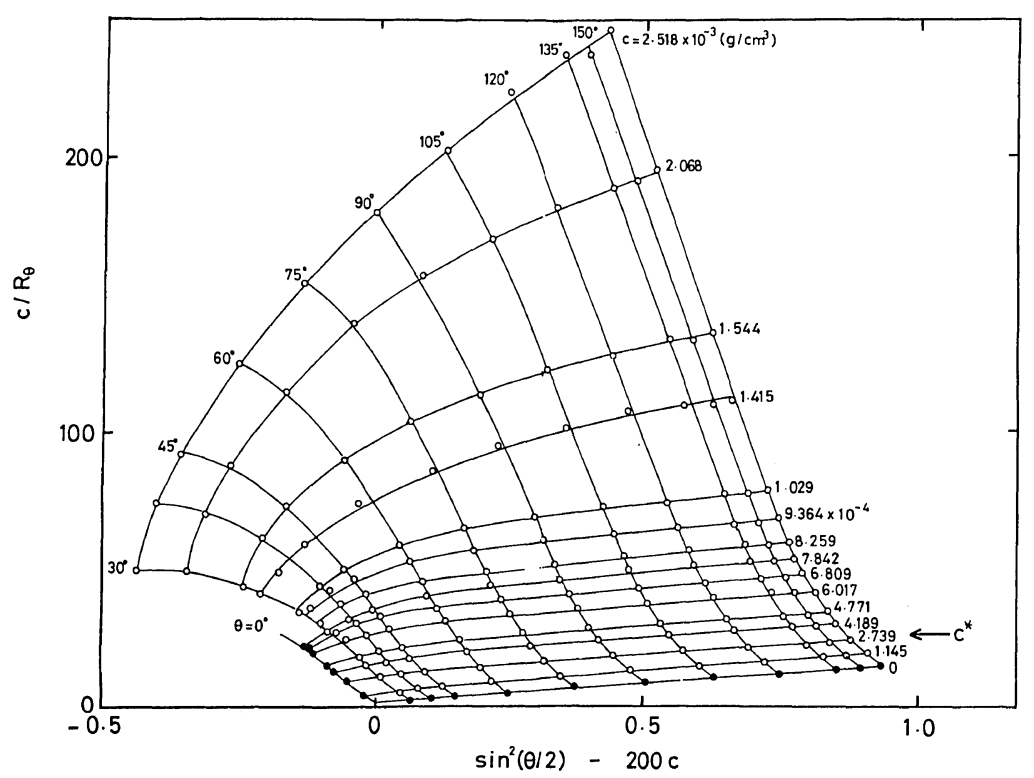

Figure 5(b). Zimm plot for PAA-0.01 M NaCl system at $25^{\circ} \mathrm{C}$.

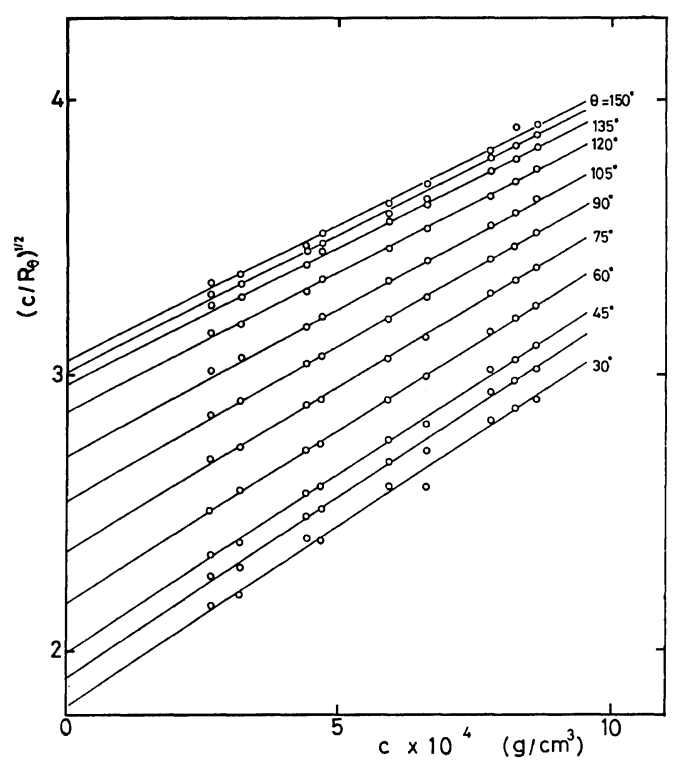

Figure 6. Square-root plot of $c / R_{\theta}$ plotted against $c$ for the PAA-0.1 M CaCl system at a dilute solution region.

where $P(\theta)$ and $Q(\theta)$ represent the intramolecular and intermolecular interference effects, respectively. In practice, we have used a square-root plot $^{15}$ instead of the Zimm plot. An example of the square- root plot against $c$ is shown in Figure 6 . The curves obtained are linear in the dilute solution region and we can get the $A_{2} Q(\theta)$ from the slope, and $A_{2}$ from the slope at $\theta=0$. However, a square-root plot against $\theta$ was convex upward more strongly than in the case of Zimm plot. This is because the $P(\theta)$ of a polyelectrolyte in solution is not represented by Debye's equation ${ }^{26}$ (eq 7), though it was shown that $P(\theta)$ of a neutral polymer of ordinary molecular weight could be represented by Debye's equation. ${ }^{27,28}$

$$
P(\theta)=\left(2 / x^{2}\right)\left(\mathrm{e}^{-x}-1+x\right)
$$

where $x=16 \pi^{2}\left\langle S^{2}\right\rangle\left[\sin (\theta / 2) / \lambda^{\prime}\right]^{2}$. By using a sharpmolecular-weight distribution sample, Nagasawa $e t$ $a .^{29}$ experimentally pointed out that $P(\theta)$ of polyelectrolyte solution obeyed Ptitsyn's equation ${ }^{30}$ (eq 8) which takes into account the excluded volume.

$$
P(\theta)=2 \int_{0}^{1}(1-y) \exp \left\{-x\left(1+\frac{5 \varepsilon}{6}+\frac{\varepsilon^{2}}{6}\right) y^{1+\varepsilon}\right\} \mathrm{d} y
$$

where $\varepsilon$ is a parameter which is zero in a $\Theta$ solvent and positive in a good solvent, $y=|i-j| / N, N$ is the number of segments in the chain, and $i$ and $j$ denote 
the $i$ th and $j$ th segment, respectively. However, we will not discuss the radius of gyration in this paper, since our sample is polydisperse.

\section{Second Virial Coefficient $A_{2}$ and Inrermolecular Interference Factor $Q(\theta)$}

The virial coefficients $A_{2}$ at different salt concentration $c_{\mathrm{s}}$ were estimated from the $\left(c / R_{\theta}\right)^{1 / 2}$ vs. $c$ plot, and are given in Table I. The values of $A_{2}$ of our experiments are $0.3-0.45$ times those of Flory. ${ }^{5}$ This is because Flory analyzed the data within a somewhat high concentration range, and hence the slope was overestimated. Even at a salt concentration as high as $c_{\mathrm{s}}=1 \mathrm{M}, A_{2}$ has a relatively high value in the order of $10^{-4}$ which is comparable to the value of a neutral polymer in a good solvent. With decrease in the salt concentration, the values of $A_{2}$ are of the order of $10^{-3}$ to $10^{-2}$ and such values are never attained in usual polymer solutions. Thus, we may expect certain characteristic behavior in the present system such as the angular dependence of $Q(\theta)$.

According to Flory, ${ }^{24}$ the concentration dependence curves of the reciprocal intensity function are not parallel but depend on the scattering angle in the Zimm plot. This becomes remarkable when the solvent becomes better. $Q(\theta)$ of poly(acrylic acid) in a $0.1 \mathrm{M} \mathrm{NaCl}$ aqueous solution is plotted against $\sin ^{2}(\theta / 2)$ by open circles in Figure 7 , and is compared with the results of polystyrene in toluene, indicated by the filled circles in Figure 7. As predicted by the theory, $Q(\theta)$ is a decreasing function of $\theta$ and reaches 0.7 in the former, while 0.9 in the latter at $\theta=150^{\circ}$. This shows that the PAA$\mathrm{NaCl}$ system behaves as a very good solvent system. Also, $Q(\theta)$ of the PAA- $0.01 \mathrm{M} \mathrm{NaCl}$ systems reaches 0.5 at $150^{\circ}$. This sort of behavior, not so pronounced in neutral polymer solutions, becomes

Table I. Second virial coefficient $A_{2}$ for poly(acrylic acid) in $\mathrm{NaCl}$ aqueous solutions at $25^{\circ} \mathrm{C}$

\begin{tabular}{cc}
\hline \multirow{2}{*}{ System } & $A_{2}$ \\
\cline { 2 - 2 } & $\mathrm{mol} \mathrm{cm}^{3} \mathrm{~g}^{-2}$ \\
\hline PAA-1 $\mathrm{M} \mathrm{NaCl}$ & $2.8 \times 10^{-4}$ \\
PAA-0.1 M NaCl & $1.3 \times 10^{-3}$ \\
PAA-0.01 M NaCl & $8.8 \times 10^{-3}$ \\
\hline
\end{tabular}

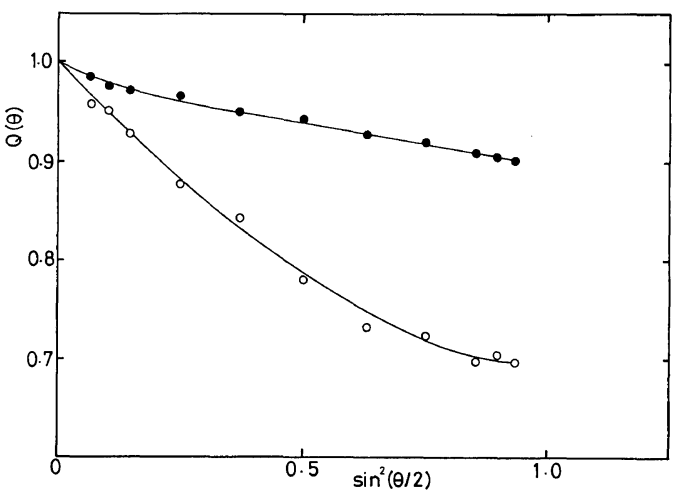

Figure 7. Angular dependence of $Q(\theta)$ : open circles, PAA-0.1 M NaCl; filled circles, polystyrene-toluene.

pronounced in a polyelectrolyte solution.

Because a polyelectrolyte solution behaves as a very good solvent system, the concentrations in our experiment cover both dilute and semi-dilute regions, as is shown more distinctly in Figure 8 wherein the phase diagram of polymer solutions is represented. Daoud and Jannink ${ }^{31}$ have reported a phase diagram for a neutral polymer solution based on a theoretical consideration which has been proved by neutron scattering. That is, the recuced temperature, $\tau=(T-\Theta) / \Theta$, in which $T$ is the temperature and $\Theta$ is the theta temperature, was plotted against the concentration $c$. This diagram is now schematically applied to aqueous salt solutions of polyelectrolytes. Because the solvent becomes better as the salt concentration for polyelectrolyte solution decreases, the solution behaves as if the temperature elevated and so $\tau$ becomes larger. The light-scattering data can be analyzed by Zimm's equation ${ }^{25}$ in those regions near the $\Theta$ temperature and in good solvents where $A_{2}$ is the order of $10^{-4}$ at the most. However, since the system under consideration here has such high values of $A_{2}$ as $10^{-3}$ $10^{-2}$, the data should be analyzed by Flory's equation ${ }^{24}$ instead of Zimm's equation described above. The increase in the $A_{2}$ value corresponds to an increase in $\tau$ as shown by (a) in Figure 8. A further increase in $A_{2}$ results in a change such as shown by (b) in the Figure, i.e., a shift to region II from region I traversing the cross over line $c^{*}$. Such a shift indicates the anomalies observed in the lightscattering curves of polyelectrolyte solutions. As an example of this, the $Z_{45} v s$. $c$ plot will be discussed below. 


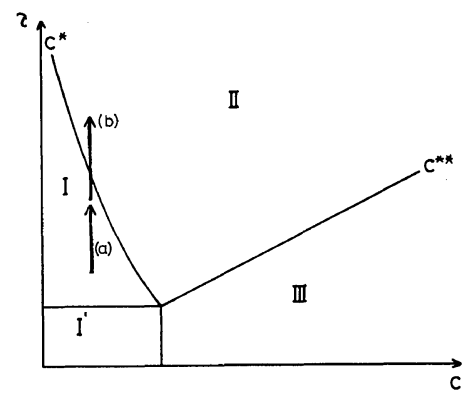

Figure 8. Phase diagram for polymer solution, in which $\tau(=(T-\Theta) / \Theta)$ is plotted against $c$. I', I, II, and III represent the $\Theta$-region, dilute region, semi-dilute region, and semi-dilute and concentrated tricritical region, respectively. $c^{*}$ is the cross-over line between I and II, and $c^{* *}$, between II and III.

\section{Concentration Dependence of Dissymmetry Ratio $Z_{45}$}

The dissymmetry ratio $Z_{45}$, a ratio of the scattered intensity at $45^{\circ}$ to that at $135^{\circ}$, usually decreases monotonously with increasing concentration. However, a minimum has been observed by Noda ${ }^{8,32}$ for polyelectrolyte solutions without or with small amounts of added salt such as $0.001 \mathrm{M}$. Now we plot $Z_{45}$ against concentration for the PAA in $1 \mathrm{M} \mathrm{NaCl}, 0.1 \mathrm{M} \mathrm{NaCl}$, and $0.01 \mathrm{M} \mathrm{NaCl}$ aqueous solutions in Figure 9. $Z_{45}$ decreases monotonously (normal behavior) at a high $\mathrm{NaCl}$ concentration, such as $1 \mathrm{M}$, but at $\mathrm{NaCl}$ concentrations of $0.1 \mathrm{M}$ and $0.01 \mathrm{M}$, a minimum appears. The polymer concentrations at the minimum point are $8.0 \times 10^{-4} \mathrm{~g} \mathrm{~cm}^{-3}$ for $0.01 \mathrm{M} \mathrm{NaCl}$ and $2.0 \times 10^{-3} \mathrm{~g} \mathrm{~cm}^{-3}$ for the $0.1 \mathrm{M} \mathrm{NaCl}$ system. The polymer concentration of $1.0-2.0 \times 10^{-4}$ $\mathrm{g} \mathrm{cm}^{-3}$ at the minimum point found by Noda at a $\mathrm{NaCl}$ concentration of $0.001 \mathrm{M}$ is about one tenth that of our result. This also shows the fact that the critical polymer concentration $c^{*}$ decreases with an decrease in the salt concentration $c_{\mathrm{s}}$, i.e., with increasing solvent power.

\section{Relation between $A_{2}$ and Microsalt Concentration}

We plotted the $A_{2}$ against $1 / c_{\mathrm{s}}$ and $1 / c_{\mathrm{s}}{ }^{1 / 2}$ in Figure 10, and found that $A_{2}$ is not proportional to $1 / c_{\mathrm{s}}{ }^{1 / 2}$ but rather to $1 / c_{\mathrm{s}}$ within the salt concentration range from $0.01-1 \mathrm{M}$. Nagasawa and Takahashi ${ }^{2,6,7}$ showed that $A_{2}$ was proportional to $1 / c_{\mathrm{s}}^{1 / 2}$ at a high salt concentration and to $1 / c_{\mathrm{s}}$ at a low salt concentration, using sodium poly(styrene

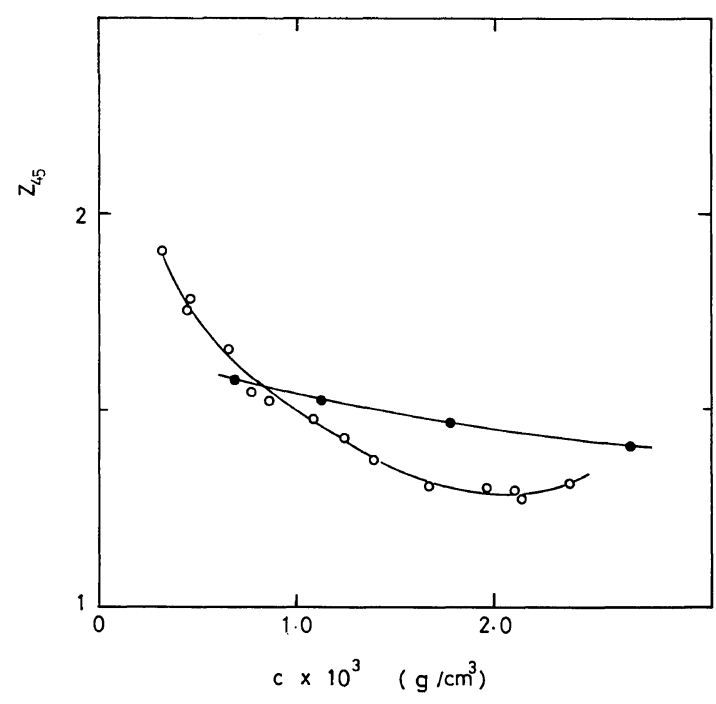

Figure 9(a). Concentration dependence of the dissymmetry ratio $Z_{45}$ : filled circles, PAA-1 $\mathrm{M} \mathrm{NaCl}$; open circles, PAA- $0.1 \mathrm{M} \mathrm{NaCl}$.

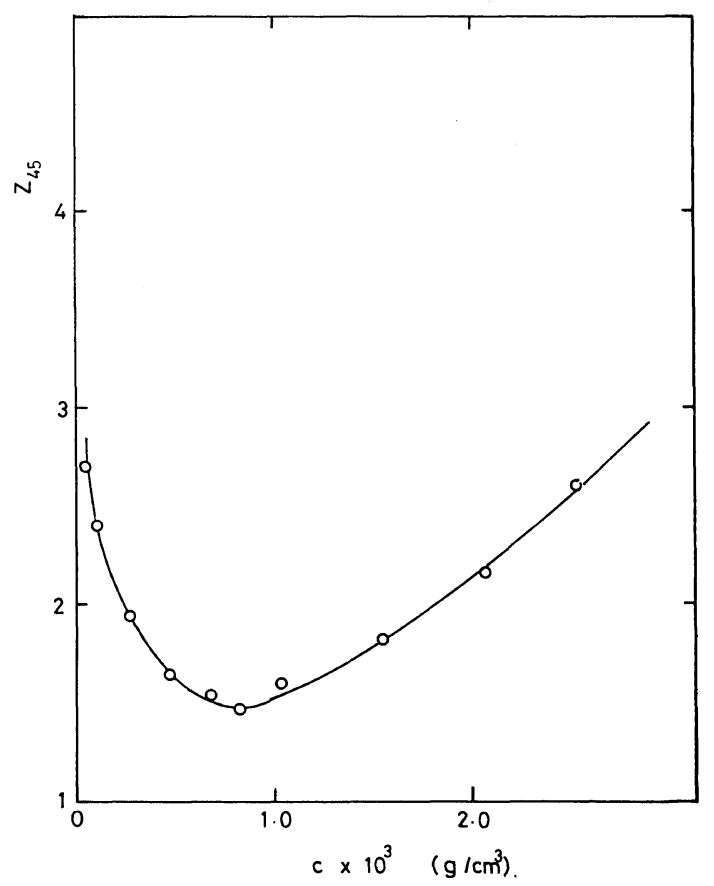

Figure 9(b). Concentration dependence of the dissymmetry ratio $Z_{45}$ for PAA- $0.01 \mathrm{M} \mathrm{NaCl}$.

sulfonate) (PSS), i.e., $A_{2} \propto 1 / c_{\mathrm{s}}{ }^{1 / 2}$ at $c_{\mathrm{s}}>0.5 \mathrm{M}$, and $A_{2} \propto 1 / c_{\mathrm{s}}$ at $c_{\mathrm{s}}<0.1-0.5 \mathrm{M}$. Because PSS is less 
Light Scattering from Polyelectrolyte in Dilute Solution

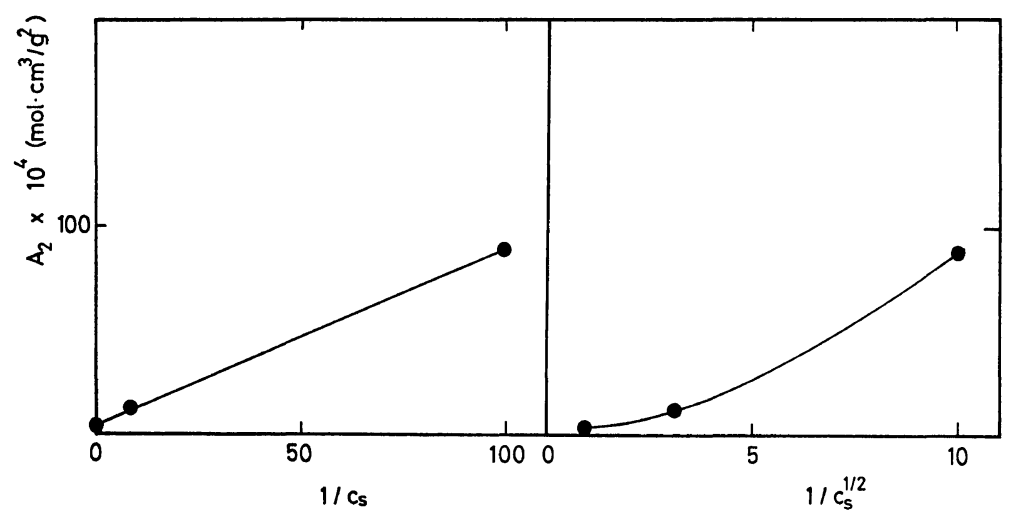

Figure 10. Plots of $A_{2}$ against $1 / c_{\mathrm{s}}$ and $1 / c_{\mathrm{s}}^{1 / 2}$.

hydrophilic than PAA, owing to its phenyl residue, it is reasonable that the upper limit of the salt concentration, where the relation $A_{2} \propto 1 / c_{\mathrm{s}}$ is valid, shifts from $0.1-0.5 \mathrm{M}$ for PSS to $1.0 \mathrm{M}$ for PAA.

Acknowledgements. The authors wish to thank Dr. Hidematsu Suzuki of Institute for Chemical Research, Kyoto University for his advice for making measurements with a Fica 50, and Doctors Akira Takahashi and Tadaya Kato of Mie University for their valuable comments and suggestions for taking measurements on polyelectrolyte solutions. Thanks are also due to Dr. Masahito Oka of our laboratory for his help in adjusting the photometer.

\section{REFERENCES}

1. S. A. Rice and M. Nagasawa, "Polyelectrolyte Solutions," Academic Press, New York, N.Y., 1961, Chapter 10.

2. A. Takahashi, T. Kato, and M. Nagasawa, J. Phys, Chem., 71, 2001 (1967).

3. M. B. Senior, S. L. H. Gorrell, and E. Hamori, Biopolymers, 10, 2387 (1971).

4. R. B. Hawkins and A. Holtzer, Macromolecules, 5, 294 (1972).

5. T. A. Orofino and P. J. Flory, J. Phys. Chem., 63, 283 (1959).

6. M. Nagasawa and A. Takahashi, "Light Scattering from Polymer Solutions" Academic Press, New York, N.Y., 1972, Chapter 16.

7. A. Takahashi, N. Kato, and M. Nagasawa, J. Phys. Chem., 74, 944 (1970).

8. A. Takahashi, "Experiment of Light Scattering" (Japanese), Nankodo, Tokyo, 1965, Chapter 6.
9. H. Utiyama, J. Phys. Chem., 69, 4138 (1965).

10. B. E. Tabor, same as ref 6, Chapter 1.

11. C. D. Thurmond, J. Polym. Sci., 8, 607 (1952).

12. D. J. Coumou, J. Colloid Sci., 15, 408 (1960).

13. G. Deželíc, J. Chem. Phys., 45, 185 (1966).

14. Data Sheet from Pressure Chemical Co. 1972.

15. G. C. Berry, J. Chem. Phys., 44, 4550 (1966).

16. T. Kotaka, N. Donkai, H. Ohnuma, and H. Inagaki, J. Polym. Sci., A-2, 6, 1803 (1968).

17. O. Bodmann, Makromol. Chem., 122, 196 (1969).

18. J. P. Kratohvil, J. Colloid Interface Sci., 21, 498 (1966).

19. Y. Tomimatsu, L. Vitello, and K. Fong, J. Colloid Interface Sci., 27, 573 (1968).

20. K. Takashima, K. Nakae, M. Shibata, and H. Yamakawa, Macromolecules, 7, 641 (1974).

21. E. F. Casassa and H. Eisenberg, Adv. Protein Chem., 19, 287 (1964).

22. H. Benoit, A. M. Holtzer, and P. Doty, J. Phys. Chem., 58, 635 (1954).

23. C. Loucheux, G. Weil, and H. Benoit, J. Chim. Phys., 55, 540 (1958).

24. P. J. Flory and A. M. Bueche, J. Polym. Sci., 27, 219 (1958).

25. B. H. Zimm, J. Chem. Phys., 16, 1093; 16, 1099 (1948).

26. P. Debye, J. Phys. Colloid Chem., 51, 18 (1947).

27. T. E. Smith and D. K. Carpenter, Macromolecules, $\mathbf{1}$, 204 (1968).

28. H. Utiyama, Y. Tsunashima, and M. Kurata, $J$. Chem. Phys., 55, 3133 (1971).

29. A. Taguchi, T. Kitano, I. Noda, and M. Nagasawa, Polym. Prepr., Jpn., 28, 508 (1979).

30. O. B. Ptitsyn, Zh. Fiz. Khim., 31, 1091 (1957).

31. M. Daoud and G. Jannink, J. Phys. (Paris), 37, 973 (1976).

32. I. Noda, Ph. D. Thesis, Nagoya University, 1963. 